\title{
Structural analysis of outer membrane beta-stranded porins using B-factor.
}

Abhishek Kumarr, S. Krishnaswamy

School of Bjotechnology, Maduraj Kamaraj University, Maduraj-625021, Tamilnadu, India

krishna@mrna.tn.nic.in Tel \#+91-452-2459141 Fax \#+91-452-2459105

\section{Introduction}

Porins are integral membrane proteins that are found in the outer membrane of Gram-negative bacteria, eukaryotic mitochondria and chloroplasts. They act as molecular sieves to ensure that the unhindered diffusion of nutrients and waste materials of size $<600 \mathrm{Da}$ into the periplasmic space while protecting the cell from hostile substances like degrading enzymes, bile salts, antibiotics, toxins, phages and abrupt changes in osmotic pressure. Porins are composed of beta barre monomers and each monomer has 250-450 residue. Typically for 16 -stranded porins, $\beta$-strands $\beta 1-\beta 5$ and $\beta 15-\beta 16$ forms the inner wall facing towards the trimer interface while $\beta 7-\beta 13$ forms the outer wall that interacts with lipids or detergents while $\beta 6$ and $\beta 14$ forms the boundary between inner and outer walls. There are loops at extracellular ends and turns at periplasmic ends. The loop L3 forms the eyelet that restricts the pore size and he loop L2 latch out with neighboring monomers. There are some other porins reported according to no of strands like 8,18 stranded porins whose crystal structures are known.

Crystallographic B-factor can be used as indicator of conformational mobility or flexibility of proteins. B-factor distribution analysis has been used earlier for analyzing structural and functional characteristics of protein structures. We have investigated the use of B-factor for analysis of mobility in some typical porin structures from the Protein Data Bank which have resolution better than $3.0 \AA$ and $R$-value $<0.23$

\section{Structures of Different porins}


R. blastica porin a 16-stranded trimeric porin which has outer (or higher) wall and a inner (lower) wall. E. coli OmpA porin a 8-stranded monomeric porin which has same convension of outer ano inner walls as of 16-stranded porins. Trimer interface of trimeric 16-stranded porins.

\section{Approach}

Selection of high resolution crystal structures $(<3.0 \AA \AA$ and R-value $<0.23)$ of porins using PDB databases.

Run the Shell Script to calculate average B-factor and corresponding standard deviation for different atom types and residue types from PDB files.

Analyse the Data with help of visualization tools

\section{Dataset}

\begin{tabular}{|r|r|r|r|r|r|r|}
\hline & Porins & PDB ID & Resolution & R-value & Length & Source \\
\hline A. & & $8-$ & Stranded & Porins & & \\
\hline 1. & OmpA & 1BXW & 2.50 & 0.19 & 171 & E.coli \\
\hline 2. & OmpX & 1 QJ8 & 1.90 & 0.20 & 148 & E.coli \\
\hline B. & & $16-$ & Stranded & Porins & & \\
\hline 1. & RBPorin & 1PRN & 1.96 & 0.18 & 289 & R. blastica \\
\hline 2. & RCPorin & 3POR & 2.5 & 0.19 & 301 & R.capsulatus \\
\hline 3. & PhoE & 1PHO & 3.0 & 0.22 & 340 & E.coli \\
\hline 4. & OmpF & 2OMF & 2.40 & 0.18 & 340 & E.coli \\
\hline
\end{tabular}

\section{Resultis}

The B-factor distributions are shown for 16-stranded porins in beta- strand (a), loops (b) and turns (c) and for 8-stranded porins both beta-strand and loops (d).
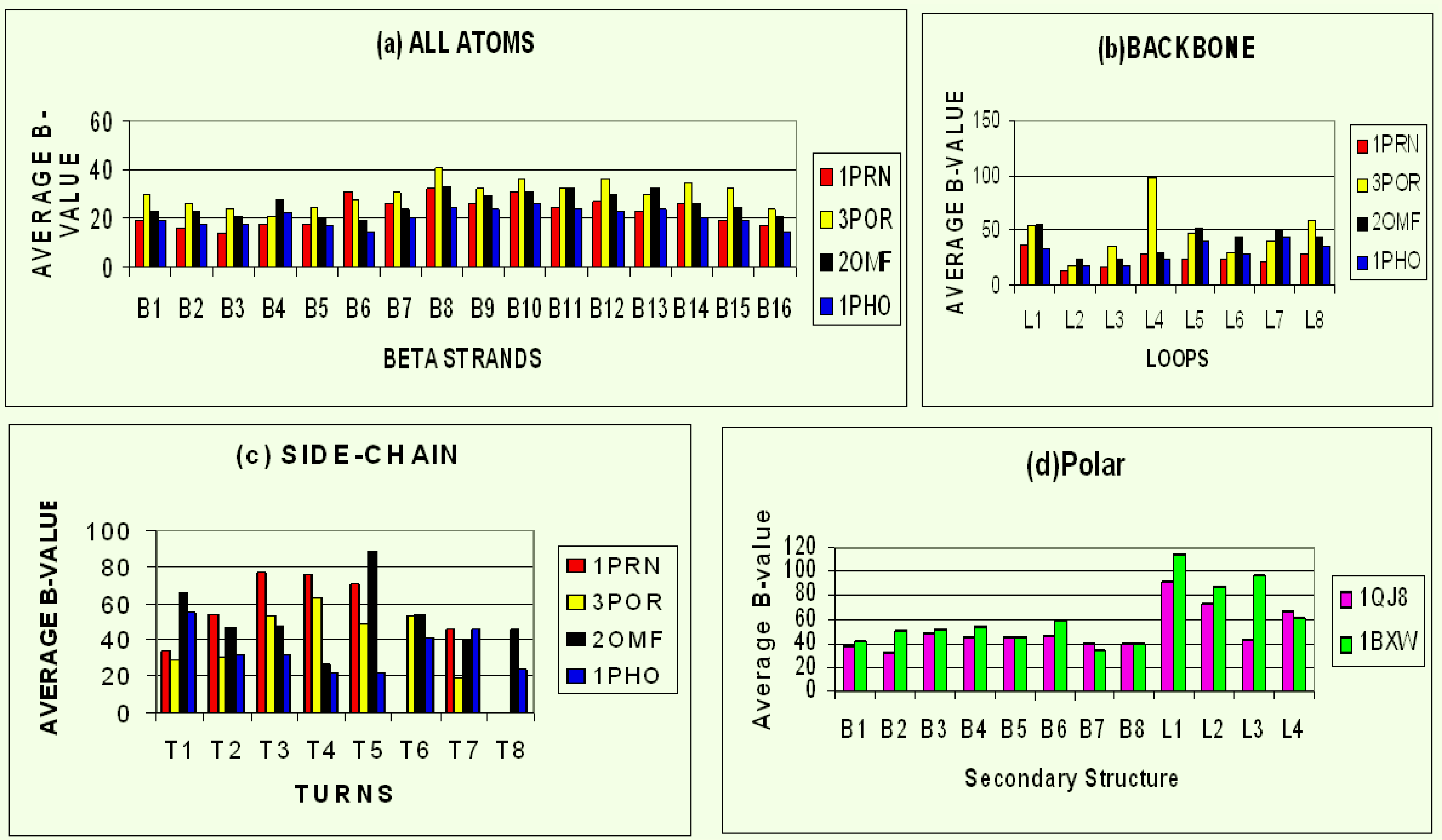

Beta strands in inner wall that is facing toward the trimer interface has lower B-values than that outer wall. For PhoE B-value is constantly lower than all porins even though it has poor resolution $(3.0 \mathrm{~A})$ amongst all four porins which infers that B-factor is intrinsic property. Loops have generally higher B-values than beta strands indicates that loops are more mobile than beta-strands. Interestingly in all porins $L 2$ and L3 have comparatively very low B-values that indicates that its functional differences from rest of the loops. Loops L5-L8 that are found in outer wall have higher mobility than others that are in inner wall. Turns show mobility as according to their occurrence. The Monomeric 8-stranded porins exhibit same pattern of mobilities as observed in 16-stranded monotrimeric porins.

There are amino acid variability amongst different porins in all secondary structure organization, example beta-strands (a). loops (b) and turns (c).

(a)CHARGE
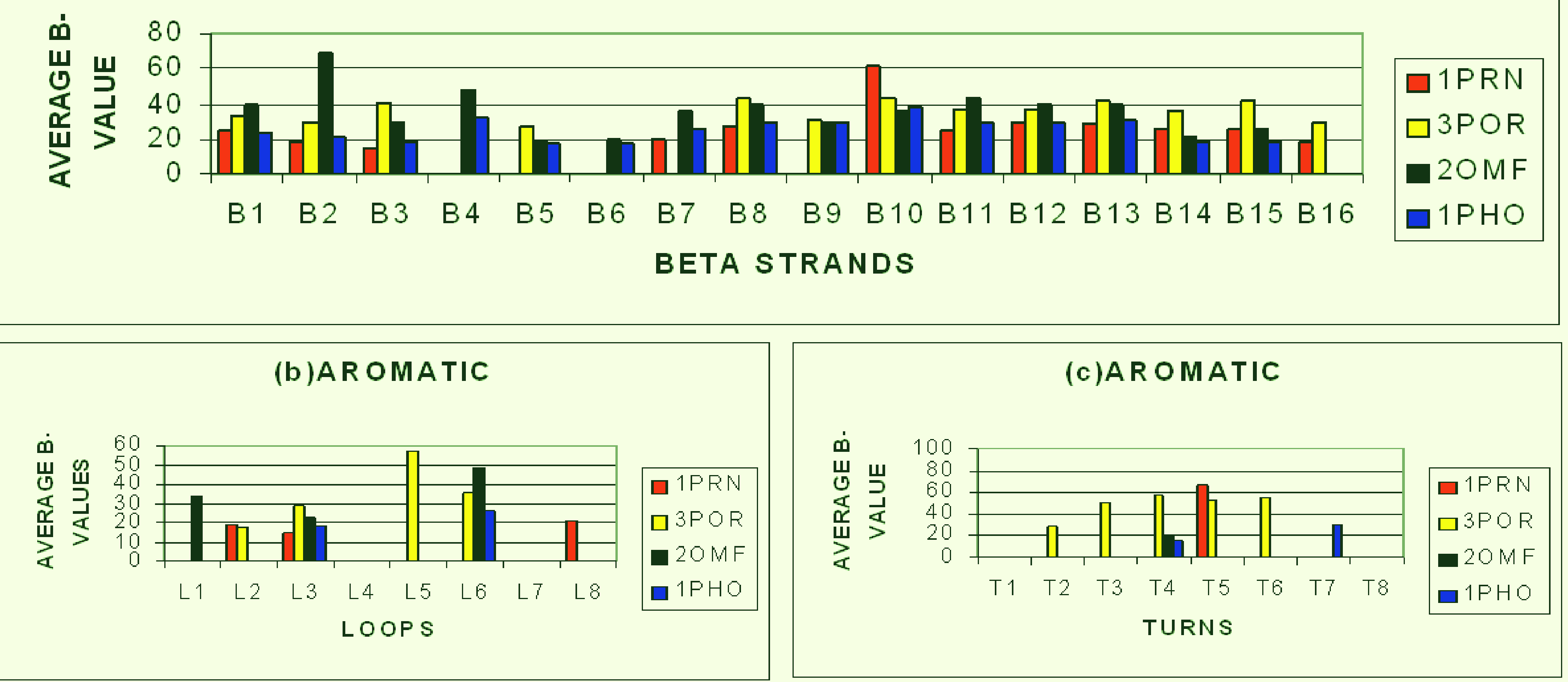

The charged residues in beta strands are varied amongst all porins, aromatic residues in loops and turns. This type of residue variability is found in all type of residue distribution analysis.

\section{Summary \& Conclusion}

*Pore is not a rigid structure.

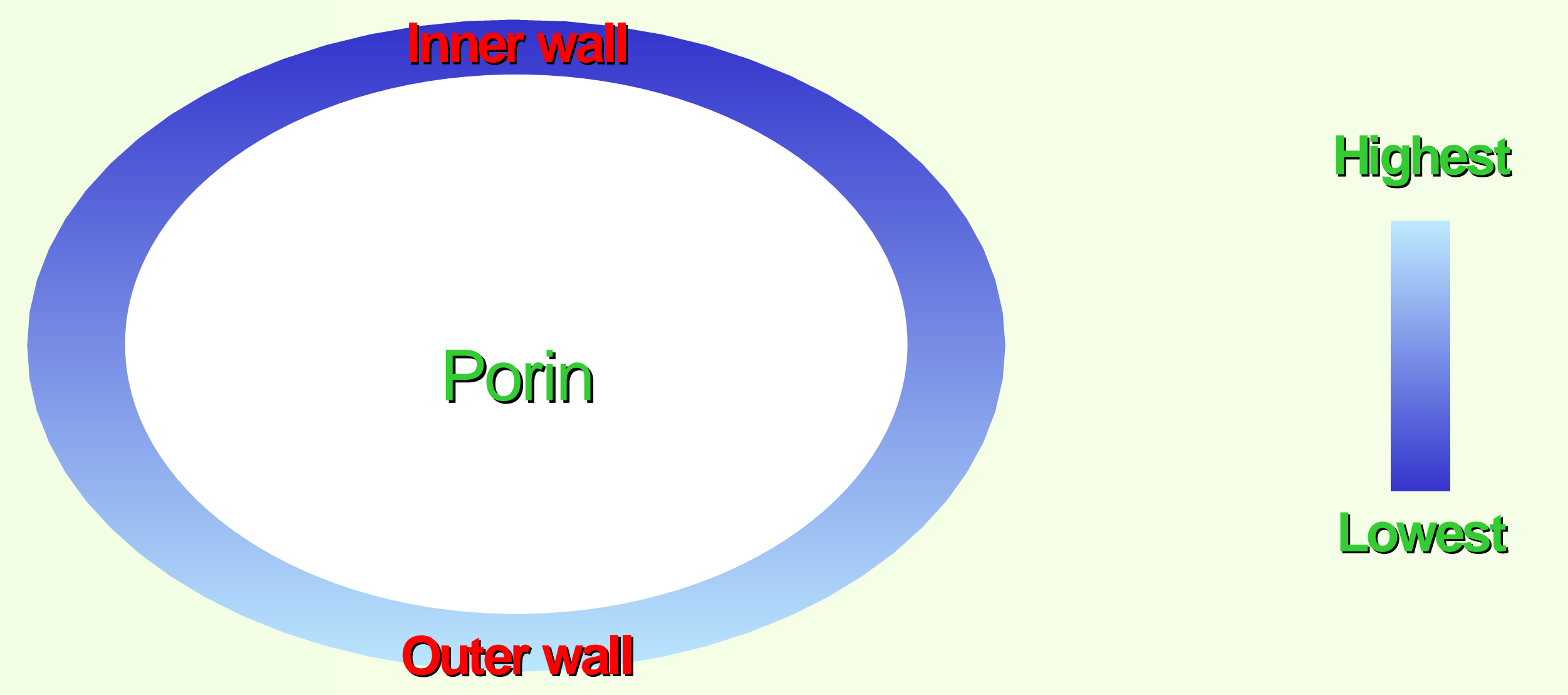

Mobility Scale

* The 16 stranded homotrimeric porins and the 8 stranded monomeric porins suggests similarity at the level of mobility of the beta strands. The outer wall has more intrinsic mobility than the inner wall suggesting that the dynamics of the pore diameter should be considered for functional characterization.

* The porins are found to be characterized by the variation in the distribution of residues and their conformational flexibility.

\section{Acknowledgements}

School of Biotechnology@Madurai Kamaraj University, India --Wonderful support

Bioinformatics Centre@Madurai Kamaraj University, India -- Facilities

Department of Biotechnology@Government of India -- Scholarship \& financial support 